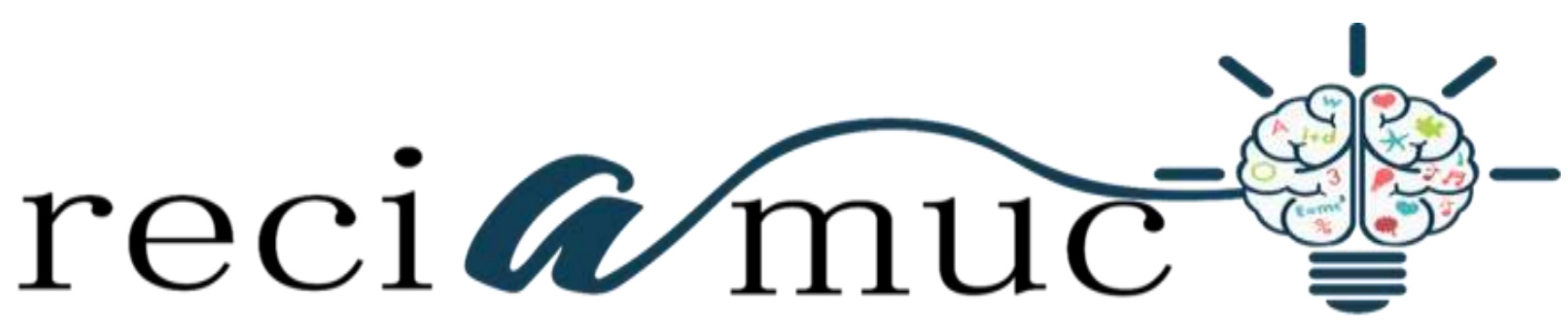

Revista científica de investigación actualización del mundo de las ciencias

Jhon Andrés González Macas a ; Evelyn Adriana Rugel Zerna ${ }^{\text {b; }}$ Pierina Yaritza Casa Gómez c; Katherine Estefania Bajaña Morán ${ }^{\mathrm{d}}$; José Luís Moncada Santillán $^{\mathrm{e}}$; Belforth Iván Vera Ganchozo ${ }^{\mathrm{f}}$

Complicaciones postoperatorias en los pacientes sometidos a cirugía de apendicitis aguda

Postoperative complications in patients undergoing surgery for acute appendicitis

Revista Científica de Investigación actualización del mundo de las Ciencias. Vol. 3 núm., 3, julio, ISSN: 2588-0748, 2018, pp. 1191-1213

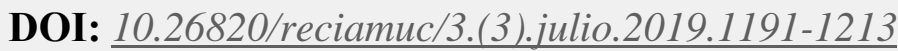

URL: $\underline{\text { http://reciamuc.com/index.php/RECIAMUC/article/view/377 }}$

Código UNESCO: 3205 Medicina Interna

Tipo de Investigación: Artículo de Investigación

(C) RECIAMUC; Editorial Saberes del Conocimiento, 2019

Recibido: 28/04/2019 Aceptado: 19/05/2019 Publicado: 01/07/2019

Correspondencia: jhonandy.gm@hotmail.es

a. Médico General en Funciones Hospitalarias; Hospital Básico Civil de Limones; jhonandy.gm@hotmail.es; Esmeralda - Ecuador.

b. Médico General en Funciones Hospitalarias; Hospital Básico Civil de Limones; earz98@ gmail.com; Esmeralda - Ecuador.

c. Médico General en Funciones Hospitalarias; Hospital Básico Civil de Limones; pierinacasag@gmail.com; Esmeralda - Ecuador.

d. Médico General en Funciones Hospitalarias; Hospital Básico Juan Carlos Guasti; kathybajanagastro@outlook.com; Esmeraldas - Ecuador.

e. Médico General; Centro de salud Puerto de Chanduy; jlmsluis@ @otmail.com; Santa Elena - Ecuador.

f. Médico Cirujano; Médico residente UCI Hospital Verdi Cevallos Balda; belforth15@ hotmail.com; Portoviejo Ecuador. 


\section{Complicaciones postoperatorias en los pacientes sometidos a cirugía de apendicitis aguda}

Vol. 3, núm. 3., (2019)

Jhon Andrés González Macas; Evelyn Adriana Rugel Zerna; Pierina Yaritza Casa Gómez;

Katherine Estefania Bajaña Morán; José Luís Moncada Santillán; Belforth Iván Vera Ganchozo

\section{RESUMEN}

En el campo de las ciencias médicas, los especialistas para llegar finalmente a un proceso quirúrgico, deben efectuar una serie de evaluaciones precisas y coherentes a la sintomatología que el paciente manifiesta. Dentro de este contexto la apendicectomía caracterizada por la inflación del apéndice, se convierte en uno de los procedimientos en medicina más común, conocido como la extracción del apéndice. Por el cirujano al momento de establecer la respectiva intervención, debe fijar su realización mediante laparoscópica, la cual responde a la utilización de instrumentos quirúrgicos especiales y una videocámara en el abdomen para extraer el apéndice. Esto permite al paciente recuperarse de manera rápida, sanar con menos dolor y cicatrices. Pero cuando, el apéndice se perforó y la infección se extendió más allá del apéndice o si tienes un absceso, es allí donde se cumple con una cirugía de apendicetomía abierta, debido a las condiciones agudas que se encuentran y se busca lograr limpiar la cavidad abdominal. Sin embargo, en estas intervenciones los pacientes van a tener diferentes complicaciones postoperatorias entre las cuales se citan: fiebre, infección de la herida, serosidad sanguinolenta, abscesos parietal e intraabdominales, hemorragia, obstrucción intestinal, fístulas estercoráceas, evisceración y eventración; situación que conduce a fijar nuevas acciones mediante la aplicación de antibióticos e incorporación de drenajes con el fin de reducir los efectos negativos agregados como resultado de la cirugía. En este sentido, la literatura expuesta, sirve de aporte referencial para el desarrollo del presente artículo cuyo propósito es analizar las complicaciones postoperatorias en los pacientes sometidos a cirugía de apendicitis aguda. Para ello, se hizo necesario seleccionar el tipo de investigación documental, acción encargada de ofrecer diferentes documentos que fueron revisados sistemáticamente para así documentar el respectivo escrito y llegar a la conclusión básica del proceso investigativo.

Palabras Claves: Complicaciones postoperatorias; pacientes; cirugía de apendicitis aguda. 


\title{
Complicaciones postoperatorias en los pacientes sometidos a cirugía de apendicitis aguda
} Vol. 3, núm. 3., (2019)

Jhon Andrés González Macas; Evelyn Adriana Rugel Zerna; Pierina Yaritza Casa Gómez; Katherine Estefania Bajaña Morán; José Luís Moncada Santillán; Belforth Iván Vera Ganchozo

\begin{abstract}
In the field of medical science, to finally reach a surgical process, specialists must be a series of accurate and consistent evaluations to the symptoms that the patient says. Within this context characterized by inflation of the Appendix, appendectomy becomes one of the most common medical procedures, known as the removal of the Appendix. By the surgeon at the time of establishing the respective intervention, you must set its realization using laparoscopic, which responds to the use of special surgical instruments and a camcorder in the abdomen to remove the Appendix. This allows the patient to recover quickly, heal with less pain and scarring. But when, the appendix was drilled and infection spread beyond appendix or if you have an abscess, it is there where it meets surgery open appendectomy, due to acute conditions that are and seeks to achieve clean the abdominal cavity. However, these interventions patients will have different postoperative complications among which are cited: fever, infection of wound, bleeding, bloody serosity, parietal and intra-abdominal abscesses, bowel obstruction, fistulas estercoraceas and evisceration, eventration; situation that leads to fix new shares through the application of antibiotics and incorporation of drains in order to reduce the negative effects added as a result of surgery. In this sense, exposed literature, serves as reference input for the development of this article whose purpose is to analyze the postoperative complications in patients undergoing surgery for acute appendicitis. To do this, it was necessary to select the type of documentary research, action responsible for offering different documents were systematically reviewed to document the respective written and reach the basic conclusions of the process investigative.
\end{abstract}

Key Words: Postoperative; Patients Complications; Acute Appendicitis Surgery. 


\section{Complicaciones postoperatorias en los pacientes sometidos a cirugía de apendicitis aguda}

Vol. 3, núm. 3., (2019)

Jhon Andrés González Macas; Evelyn Adriana Rugel Zerna; Pierina Yaritza Casa Gómez; Katherine Estefania Bajaña Morán; José Luís Moncada Santillán; Belforth Iván Vera Ganchozo

\section{Introducción.}

La apendicitis es un proceso evolutivo, secuencial, de allí las diversas manifestaciones clínicas y anatomopatológicas que suele encontrar el cirujano, las cuales dependerán fundamentalmente del momento o fase de la enfermedad en que es abordado el paciente, de allí que se consideren los siguientes estadios: apendicitis congestiva o catarral, apendicitis flemonosa o supurativa, apendicitis gangrenosa o necrótica, apendicitis perforada. En consecuencia, la apendicectomía sigue siendo uno de los procedimientos de emergencia más comunes realizados por los cirujanos gastrointestinales., teniendo una mayor incidencia la apendicitis aguda.

Cabe destacar que, la apendicitis se refiere a la inflamación del apéndice, siendo la apendicectomía, la extracción quirúrgica del apéndice, que se realiza principalmente en pacientes que presentan apendicitis aguda. Los cuales son sometidos a una apendicectomía para la apendicitis complicada, que se define como apendicitis gangrenosa (muerte del tejido blando) o perforada (rotura), tienen mayor probabilidad de sufrir complicaciones posoperatorias. Ante lo cual, se hace la colocación sistemática de un drenaje quirúrgico para prevenir el absceso intraperitoneal (una colección localizada de pus en el abdomen o la pelvis) después de una apendicectomía para la apendicitis complicada es polémica.

En consecuencia, una vez definido el diagnóstico de la apendicitis aguda debe efectuarse la apendicectomía, por cuanto los resultados de la intervención quirúrgica se hallan relacionados con la precocidad de esta última y con la evolución del proceso inflamatorio apendicular. Este proceder está indicado, tanto en los casos confirmados, como en los que la misma no pueda ser excluida del diagnóstico diferencial, puesto que la morbilidad y mortalidad ocasionadas por una exploración del abdomen, bien a través de la laparotomía convencional o de la video laparoscópica, son, apreciablemente, menores que las producidas al dejar evolucionar un apéndice inflamado hacia sus fases más graves (gangrenosa y perforada), sobre todo en niños y ancianos.

No obstante, la infección de la herida es casi la mitad de probable después de la apendicectomía aguda; pues, los abscesos intraabdominales ocurren casi 3 veces más frecuentemente. La razón 


\section{Complicaciones postoperatorias en los pacientes sometidos a cirugía de apendicitis aguda}

Vol. 3, núm. 3., (2019)

Jhon Andrés González Macas; Evelyn Adriana Rugel Zerna; Pierina Yaritza Casa Gómez; Katherine Estefania Bajaña Morán; José Luís Moncada Santillán; Belforth Iván Vera Ganchozo

para este aparente aumento en la formación de abscesos después de la intervención quirúrgica. Una de las explicaciones es la presencia de cuerpos extraños que causan obstrucción, produciéndose una disminución en la oxigenación en el tejido del apéndice, ulceración y posterior invasión de bacterias. Por ello, el método de diagnóstico más importante de apendicitis aguda, es la evaluación realizada por el médico, quien a partir del examen físico realizado al paciente e interrogatorio acerca del dolor y síntomas asociados, puede sospechar la causa del cuadro clínico, para descartar otro tipo de entidades. Los pacientes con diagnóstico de apendicitis aguda deben ser hospitalizados y evaluados por un especialista en cirugía, quien realizará el manejo quirúrgico.

En este sentido, Sample (2015) precisa que las complicaciones de las intervenciones por apendicitis aguda:

No son infrecuentes, a pesar de los avances tecnológicos de la cirugía, anestesiología y reanimación, reducen al mínimo el traumatismo operatorio, de la existencia de salas de cuidados intensivos y cuidados intermedios, para la atención de pacientes graves y de que hoy se utilizan antibióticos cada vez más potentes. Pero se encuentran entre estas complicaciones las siguientes: serosidad sanguinolenta en la herida quirúrgica por cuerpo extraño o hemostasia deficiente, abscesos parietal e intraabdominal, hemorragia, flebitis, epiploítis, obstrucción intestinal, fístulas estercoráceas, evisceración y eventración. (p.8)

$\mathrm{Al}$ analizar el respectivo aporte, se puede entender que debido a los avances científicos en las áreas médicas complementados por los eventos tecnológicos, conduce a reducir las complicaciones; pero debido a las características fisiopatológicas de los pacientes, se van a registrar la presencia de diferentes complicaciones que ameritan la intervención clínica, con el fin de reducir estos efectos, para ello, se incorpora tratamiento a través de una selección de antibióticos que están estrechamente relacionados con las condiciones generales del paciente, además utilizan en algunos casos el drenaje

Este mismo autor, precisa que en los últimos 50 años ha ocurrido un dramático descenso de la mortalidad asociada a la apendicitis aguda (desde 26,0 hasta menos del 1,0 \%). Sin embargo, la 


\section{Complicaciones postoperatorias en los pacientes sometidos a cirugía de apendicitis aguda}

Vol. 3, núm. 3., (2019)

Jhon Andrés González Macas; Evelyn Adriana Rugel Zerna; Pierina Yaritza Casa Gómez; Katherine Estefania Bajaña Morán; José Luís Moncada Santillán; Belforth Iván Vera Ganchozo

morbilidad todavía hoy refleja una alta incidencia de perforaciones $(17,0$ al 20,0 \%) a pesar de los diagnósticos realizados por imágenes y del desarrollo biotecnológico alcanzado. Asimismo, complementa que el riesgo de apendicitis después de los 50 años es de 1:35 para mujeres y 1:50 para varones, su morbimortalidad de apendicitis aguda en este grupo de pacientes continúa elevada.

Según las consideraciones anteriores, se precisa que la apendicetomía por apendicitis aguda continúa siendo hoy la operación de urgencia más frecuente en los servicios quirúrgicos y, a pesar de eso, las causas que condicionan sus complicaciones son aún poco conocidas, por lo que su determinación sería importante disminuir la morbilidad y mortalidad postoperatorias. La misma, es una patología de adultos jóvenes, el aumento de la expectativa de vida en las personas mayores de 65 años contribuye a que el médico cirujano diagnostique de manera más frecuente esta patología en este grupo etario.

En esta misma dirección, Medina (2017) indica que al igual que en el adulto, el niño que presenta la apendicitis aguda es la:

Patología quirúrgica de urgencia más frecuentemente diagnosticada y constituye un desafío diagnóstico debido a la superposición de síntomas con otras patologías especialmente en los menores de 4 años. El retraso en su reconocimiento se asocia a un aumento de la morbilidad, mortalidad y costos médicos. Solo el $2 \%$ de las apendicitis se presenta en menores de 3 años y en ellos la presentación clínica más frecuente es atípica por lo que el diagnóstico es tardío. La incidencia desde el nacimiento hasta los cuatro años es en promedio de 1 a 2 en 10000 niños por año. (p.11)

De acuerdo con este planteamiento, se precisa que la tendencia a diferentes complicaciones existentes una vez realizada la intervención quirúrgica de apendicitis aguda, va a estar correlacionada con el diagnóstico primario que el médico realice, pues, una oportuna identificación de los síntomas y reducción de relacionarse con otras patologías, hace posible reducir la presencia de complicaciones postoperatorias, dado que, la herida no acumulará bacterias que hagan posible 


\section{Complicaciones postoperatorias en los pacientes sometidos a cirugía de apendicitis aguda}

Vol. 3, núm. 3., (2019)

Jhon Andrés González Macas; Evelyn Adriana Rugel Zerna; Pierina Yaritza Casa Gómez; Katherine Estefania Bajaña Morán; José Luís Moncada Santillán; Belforth Iván Vera Ganchozo

la presencia de microorganismos y conduzcan a la presencia de complicaciones que ameriten una atención médica especializada.

En consecuencia, con el fin de reducir las posibles complicaciones postoperatorias de una intervención de apendicitis aguda, es relevante considerar la profilaxis antibiótica, la misma puede ser recomendada pre o postoperatoriamente y deberá ser considerada para su uso de rutina en la apendicetomía de emergencia. Mediante su utilización se reducen las complicaciones infecciosas en estos pacientes intervenidos. Dado que, las complicaciones que puedan manifestar los pacientes en relación a la cicatrización post operatoria de una apendicitis, las mismas son posibles eventualidades en el curso del procedimiento quirúrgico con una respuesta local o sistémica que puede retrasar la recuperación del paciente o poner en riesgo su vida. Por ello, el uso de los antibióticos, es importante, mediante su manejo efectivo, se logra una mejoría en las condiciones de salud. Asimismo, el médico puede, al realizar una evaluación de la herida y al encontrar pus o secreción, mal olor, fiebre, enrojecimiento y dolor al tocar, es importante recurrir al uso del drenaje como una alternativa que ayuda a la expulsión de la secreción o pus.

Cada uno de los planteamientos citados en párrafos anteriores, son los encargados de direccionar la conducción metodológica del presente artículo, mediante su construcción se busca llegar a un análisis de las complicaciones postoperatorias en los pacientes sometidos a una cirugía de apendicitis aguda, de allí, la significación de generar diferentes apreciaciones relativas al tema, con el fin de propiciar nuevas ideas encargadas de logar el respectivo reconocimiento de aquellos agentes encargados de manifestar complicaciones postoperatorias que puedan afectar la salud o vida del paciente, es allí, donde la actuación oportuna del médico hace posible reconocer las situaciones que presenta el paciente y reducir los efectos negativos que pudieran hacerse presente luego de la apendicectomía aguda.

\section{Método.}

Los aspectos que conforman este apartado se encuentran determinados por una serie de elementos dirigidos a reconocer el camino metodológico del estudio, para así especificar sus características generales y fijar posiciones claras en cuanto al método, tipo de investigación, fuentes documentales 


\section{Complicaciones postoperatorias en los pacientes sometidos a cirugía de apendicitis aguda}

Vol. 3, núm. 3., (2019)

Jhon Andrés González Macas; Evelyn Adriana Rugel Zerna; Pierina Yaritza Casa Gómez; Katherine Estefania Bajaña Morán; José Luís Moncada Santillán; Belforth Iván Vera Ganchozo

y técnicas de recolección de la información. En cuanto al método, se selecciona inductivo, definido por Méndez (2018) "es el proceso de razonamiento de una parte de un todo, va de lo particular a lo general, de lo individual a lo universal". (p.44). Es decir, mediante la incorporación de este método, se logra desglosar en forma individual los aspectos que conforman el tema seleccionado, para luego agrupar las ideas y formular acciones innovadoras que aseguren interpretaciones válidas para todos los lectores.

Según la definición dada, se puede considerar que el desarrollo del artículo amerita, de separar las consideraciones para convertirlas en acciones reales que puedan ser comprendidas por diferentes grupos interesados en la temática y así, asegurar su expansión en el campo de las ciencias médicas, ambientes familiares u otros grupos, interesados por descifrar algunas interrogantes relativas a las complicaciones postoperatorias en los pacientes sometidos a una cirugía de apendicitis aguda para tomar posiciones viables.

\section{Tipo de Investigación.}

El desarrollo de la presente investigación, corresponde al tipo documental, definida por Mogollón (2019) como aquella que está "basada en nuevos conocimientos con el objetivo de aumentar los ya existentes sin utilizar aplicaciones prácticas, sólo documentaciones que sirva como material de estudio". (p.64). Por ello, su conducción a través de este tipo de estudio, podrá hacer posible dirigir un proceso de revisión en diferentes materiales impresos, tecnológicos que permitan con el apoyo del método inductivo, individualizar los criterios para luego convertirlos en hechos universales.

Otra definición considerada para argumentar el presente artículo, son el ofrecido por, Castellanos (2018) "mediante la investigación documental se puede organizar de forma flexible diferentes criterios establecidos y convertirlos en nuevas directrices para propiciar verdaderos cambios en un documento científico e interpretativo". (p.32). Es decir, el adecuar los pasos que estructuran el camino metodológico del proceso científico, significa ubicar el trabajo dentro de una conceptualización que ofrezca nuevas formas de direccionar el hacer, en otras palabras, ayudar al investigador a construir sus ideas tomando como punto de referencia los aportes de otros criterios. 


\section{Complicaciones postoperatorias en los pacientes sometidos a cirugía de apendicitis aguda}

Vol. 3, núm. 3., (2019)

Jhon Andrés González Macas; Evelyn Adriana Rugel Zerna; Pierina Yaritza Casa Gómez; Katherine Estefania Bajaña Morán; José Luís Moncada Santillán; Belforth Iván Vera Ganchozo

\section{Fuentes Documentales.}

Con el fin de dar continuidad al proceso investigativo, es importante ubicarse en aquellas fuentes documentales que sirven de aporte para la ubicación y selección del material impreso y tecnológico vinculado con el tema previamente seleccionado, Por ello, para cumplir dichas actividades se tuvo la necesidad de implementar métodos ajustados a las condiciones documentales que caracterizan al trabajo, dentro de ellos, se incluyen el deductivo mediante el cual, se lograron interpretar las ideas encontrada en los textos o material bibliográfico desde una visión general, para llegar a establecer argumentaciones parciales o particulares. Tal como lo asevera Castellanos (ob.cit) "representadas por aquellos informes transcritos que sirven para ampliar de manera precisa el contenido de un tema." (p.41).

En consecuencia, su utilización permite llegar a la revisión de los diferentes artículos, revistas, libros entre otros que responde a la naturaleza de la temática en el proceso investigativo, aunado a la complementación que dan los diversos textos médicos para ampliar las apreciaciones pertinentes. En tal sentido, Castellanos (ob.cit), "representan los aportes bibliográficos para el desarrollo de la temática previamente seleccionada y transformarla en un acto investigativo real". (p.74)

\section{Técnicas para la Recolección de la Información.}

En cuanto a las técnicas empleadas se destacan el fichaje mediante el cual, se organizaron los diversos textos de acuerdo a la necesidad de las fuentes primarias requeridas para el desarrollo de los contenidos a tratar en la investigación. Otra técnica de importancia fue el resumen, mediante ella, se desarrollaron aquellos elementos particulares extraídos de textos generales que luego al ser incorporados al argumento general del estudio, hicieron posible su complementación teórica. Cada uno de los pasos fijados, llevan a resaltar que el análisis correspondiente se cumple, mediante la introducción de aquellos métodos descriptivos e interpretativos, capaces de llevar a la autora hacia la construcción de diferentes argumentos consistentes en sus valoraciones como resultado a la lectura previa de los documentos requeridos para el desarrollo del proceso investigativo. 


\section{Complicaciones postoperatorias en los pacientes sometidos a cirugía de apendicitis aguda}

Vol. 3, núm. 3., (2019)

Jhon Andrés González Macas; Evelyn Adriana Rugel Zerna; Pierina Yaritza Casa Gómez;

Katherine Estefania Bajaña Morán; José Luís Moncada Santillán; Belforth Iván Vera Ganchozo

Cabe agregar, que cada uno de los elementos incorporados en el proceso de indagación, hacen posible abrir paso a las primeras actividades inherentes a la selección del tema, revisión de la literatura pertinente, para luego organizar, subrayar e interpretar sus contenidos de manera reflexiva y hacer de ello aportes relevantes que dan cabida a nuevas valoraciones ideales estrechamente relacionadas con el contenido temático, para asegurar la efectividad del mismo al momento de su inclusión en el campo de las ciencias médicas. Es decir, el trabajo científico para obtener resultados significativos, debe ser guiado por el desarrollo de técnicas básicas que aseguren con anticipación la construcción de un reportaje eficiente para todos los lectores interesados en su contenido.

\section{Resultados.}

El proceso investigativo relacionado con la revisión bibliográfica, llevada a cabo, permite construir diferentes aportes significativos relacionados con los elementos caracterizados por el desarrollo de los eventos propios que estructuran el contenido del artículo hacia una dirección metodológica previamente preestablecida, entre ellos se encuentran a continuación los siguientes argumentos.

\section{Etiología, Patogenia y Anatomopatológico de la Apendicitis Aguda.}

El factor etiológico predominante en la apendicitis aguda es la obstrucción de la luz. Los fecalitos son la causa común de la obstrucción apendicular. Menos frecuentes son la hipertrofia de tejido linfoide, impacto de bario por estudios radiológicos previo, tumores, semillas de verduras y frutas y parásitos intestinales. La frecuencia de obstrucción aumenta con la gravedad del proceso inflamatorio. Al respecto, Arroyo (2017), indica que se reconocen fecalitos en $40 \%$ de los casos de apendicitis aguda simple, en $65 \%$ de la apendicitis gangrenosa sin rotura y en casi $90 \%$ de los pacientes con apendicitis gangrenosa con rotura." (p.25)

Según lo citado, existe una secuencia predecible de acontecimientos que conduce a la rotura final del apéndice. La obstrucción proximal de la luz apendicular provoca una obstrucción de asa cerrada y la continuación de la secreción normal por la mucosa apendicular da lugar a una rápida distensión. La distensión del apéndice estimula terminaciones nerviosas de fibras viscerales 


\section{Complicaciones postoperatorias en los pacientes sometidos a cirugía de apendicitis aguda}

Vol. 3, núm. 3., (2019)

Jhon Andrés González Macas; Evelyn Adriana Rugel Zerna; Pierina Yaritza Casa Gómez; Katherine Estefania Bajaña Morán; José Luís Moncada Santillán; Belforth Iván Vera Ganchozo

aferentes de estiramiento y causa dolor vago, sordo y difuso en el abdomen medio o el epigastrio bajo.

Asimismo, se estimula el peristaltismo por la distensión bastante súbita, de tal manera que al inicio del curso de la apendicitis pueden superponerse algunos cólicos al dolor visceral. La distensión aumenta por la continuación de la secreción mucosa y la multiplicación rápida de las bacterias que residen en el apéndice. La distensión de esta magnitud suele causar náuseas y vómitos reflejos, y el dolor visceral difuso se torna más intenso. A medida que asciende la presión en el órgano, se excede la presión venosa. Se ocluyen capilares y vénulas, pero continúa el flujo arteriolar de entrada, lo que da por resultado ingurgitación y congestión vascular. El proceso inflamatorio incluye en poco tiempo la serosa del apéndice y el peritoneo parietal de la región, lo cual suscita el cambio característico del dolor hacia el cuadrante inferior derecho.

En otras palabras, la mucosa del tubo digestivo, incluido el apéndice, es susceptible a un deterioro del riego y en consecuencia se altera temprano su integridad en el proceso, lo que promueve una invasión bacteriana. Conforme la distensión progresiva afecta primero el retorno venoso y luego el flujo de entrada arteriolar, sufre más el área con la irrigación más deficiente: se desarrollan infartos elipsoidales en el borde antimesentérico. Por lo regular, la perforación tiene lugar justo después del punto de obstrucción, no tanto en la punta por el efecto del diámetro sobre la tensión intraluminal. 


\section{Complicaciones postoperatorias en los pacientes sometidos a cirugía de apendicitis aguda}

Vol. 3, núm. 3., (2019)

Jhon Andrés González Macas; Evelyn Adriana Rugel Zerna; Pierina Yaritza Casa Gómez; Katherine Estefania Bajaña Morán; José Luís Moncada Santillán; Belforth Iván Vera Ganchozo

\section{Imagen $\mathbf{N}^{\circ} 1$ Ubicación de la Apéndice}

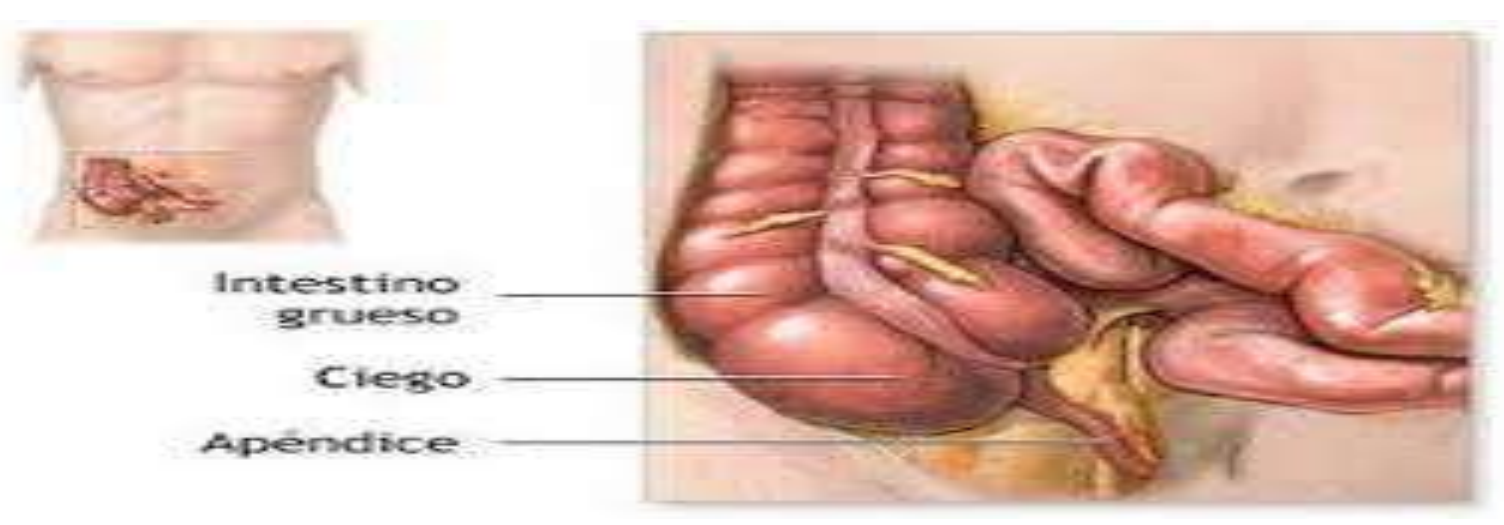

Fuente: Arroyo (ob.cit)

Según la imagen que antecede, se puede observar que, al hacer de apendicitis, no es más que un proceso evolutivo, secuencial, de allí las diversas manifestaciones clínicas y anatomopatológicas que suele encontrar el cirujano y que dependerán fundamentalmente del momento o fase de la enfermedad en que es abordado el paciente, de allí que se consideren los siguientes estadios, desarrollados por el autor citado:

Apendicitis Congestiva o Catarral: caracterizada por cuando ocurre la obstrucción del lumen apendicular se acumula la secreción mucosa y agudamente distiende el lumen. El aumento de la presión intraluminal produce una obstrucción venosa, acúmulo de bacterias y reacción del tejido linfoide, que produce un exudado plasmoleucocitario denso que va infiltrando las capas superficiales. Todo esto macroscópicamente se traduce en edema y congestión de la serosa.

Apendicitis Flemonosa o Supurativa: la mucosa comienza a presentar pequeñas ulceraciones o es completamente destruida siendo invadida por enterobacterias, coleccionándose un exudado mucopurulento en la luz y una infiltración de leucocitos neutrófilos y eosinófilos en todas las túnicas incluyendo la serosa, que se muestra intensamente congestiva, edematosa, de coloración rojiza y con exudado fibrino-purulento en su superficie; si bien aún no hay perforación de la pared apendicular, se produce difusión del contenido mucopurulento intraluminal hacia la cavidad libre. 


\section{Complicaciones postoperatorias en los pacientes sometidos a cirugía de apendicitis aguda}

Vol. 3, núm. 3., (2019)

Jhon Andrés González Macas; Evelyn Adriana Rugel Zerna; Pierina Yaritza Casa Gómez; Katherine Estefania Bajaña Morán; José Luís Moncada Santillán; Belforth Iván Vera Ganchozo

Apendicitis Gangrenosa o Necrótica: cuando el proceso flemonoso es muy intenso, la congestión y la distensión del órgano producen anoxia de los tejidos, a ello se agrega la mayor virulencia de las bacterias y a su vez el aumento de la flora anaeróbica, que llevan a una necrobiosis total. La superficie del apéndice presenta áreas de color púrpura, verde gris o rojo oscuro, con microperforaciones, aumenta el líquido peritoneal, que puede ser tenuamente purulento con un olor fecaloideo.

Apendicitis Perforada: cuando las perforaciones pequeñas se hacen más grandes, generalmente en el borde antimesentérico y adyacente a un fecalito, el líquido peritoneal se hace francamente purulento y de olor fétido. Siendo más común en los adultos mayores. Las tasas de perforación aumentan en forma directamente proporcional con la edad. En una serie se encontró perforación en $47 \%$ de los sujetos de 60 a 64 años, en $69 \%$ de los enfermos de 70 a 74 años y en $71 \%$ de los que tenían entre 80 y 84 años de edad. Otros informan tasas de perforación de hasta 90\% para sujetos mayores de 70 años de edad. Todavía no existe un acuerdo de cuál es la causa primordial en la alta incidencia de perforación en pacientes adultos mayores.

Cabe destacar que, la presencia de los diferentes tipos de apendicitis, las cuales tienen como característica final la respectiva intervención quirúrgica, pues, se hace notorio, la presencia de una sintomatología caracterizada por el dolor periumbilical que se localiza por un periodo de varias horas en el cuadrante inferior derecho, es el principal síntoma. En el adulto mayor, que tiene un umbral más elevado para este síntoma, adquiere una menor expresividad clínica, el dolor abdominal tiende a ser vago y difuso. Además, puesto que el dolor abdominal vago es una manifestación común en personas de edad avanzada, a menudo se pasa por alto su importancia.

Además, la contractura muscular refleja también suele ser menos marcada que en el adulto y en el niño, e incluso, puede faltar en presencia de peritonitis. Esto se explica por la frecuente flacidez de la pared abdominal en los pacientes de edad avanzada. También, se hace evidente los vómitos, como expresión de insuficiencia muscular y de depresión de las actividades reflejas, sean substituidos por náuseas, escalofríos y fiebre, generalmente frecuentes en algunas formas de abdomen agudo, suelen ser poco intensos o pueden no producirse en los adultos mayores. 


\section{Complicaciones postoperatorias en los pacientes sometidos a cirugía de apendicitis aguda}

Vol. 3, núm. 3., (2019)

Jhon Andrés González Macas; Evelyn Adriana Rugel Zerna; Pierina Yaritza Casa Gómez; Katherine Estefania Bajaña Morán; José Luís Moncada Santillán; Belforth Iván Vera Ganchozo

De lo antes planteado, se precisa que clínicamente, el cuadro clásico de apendicitis aguda aparece sólo en una cuarta parte de los pacientes. Muchas veces la clínica es atípica: dolor de comienzo, topografía e intensidad muy variables, aunque frecuentemente el dolor termina localizándose en la fosa ilíaca derecha después de un período evolutivo mayor que en los jóvenes. Los hallazgos del examen físico son variables: distensión abdominal, signos de irritación peritoneal, silencio abdominal a la auscultación, masa palpable en cuadrante inferior derecho del abdomen.

En general, el cuadro clínico tiene un comienzo más larvado, con síntomas atenuados, por lo que los errores diagnósticos son frecuentes, no sólo por el cuadro clínico atípico, sino también porque no siempre se piensa que una apendicitis aguda es la responsable del cuadro clínico de abdomen agudo en los adultos mayores. Los hallazgos de laboratorio tampoco son fiables en los adultos mayores. Los recuentos leucocitarios son menores de 10000 células/mm3 en 20 a 50\% de los adultos de edad avanzada con apendicitis simple.

Es importante destacar que, la ultrasonografía es un procedimiento no invasivo y tiene un índice de precisión de más del $80 \%$ aunque es poco sensible para casos no típicos. La visualización de un apéndice aumentado de tamaño (más de $6 \mathrm{~mm}$ de diámetro) y de pared gruesa es muy sugerente de una apendicitis aguda, con la limitación de que en ocasiones no va a ser posible localizar el apéndice. Actualmente, la técnica de mayor precisión es la tomografía computarizada (TAC). Posee una sensibilidad y especificidad del 90 y 95\%, respectivamente. Tiene una precisión mayor del 90\%, superior a la de la ecografía, debido a que identifica mejor el apéndice y porque, además, detecta los cambios inflamatorios periapendiculares.

Cuadro $\mathbf{N}^{\circ} 1$ Microorganismos Comunes en Apendicitis Aguda

\begin{tabular}{|c|c|}
\hline Aerobios y Facultativos & Anerobios \\
\hline Bacilos gramnegativos & Bacilos Gram Negativos \\
E coli & Bacteroides fragilis \\
\hline Pseudomonas aeuroginosa & Otras especies de Bacteroides \\
Especies de Klebsiella & Especies de Fusobacterium \\
\hline Cocos Gram positivos & Cocos Gram Positivos \\
Streptucoccus anginosus & Especies de Peptostreptococus \\
\hline
\end{tabular}




\section{Complicaciones postoperatorias en los pacientes sometidos a cirugía de apendicitis aguda}

Vol. 3, núm. 3., (2019)

Jhon Andrés González Macas; Evelyn Adriana Rugel Zerna; Pierina Yaritza Casa Gómez; Katherine Estefania Bajaña Morán; José Luís Moncada Santillán; Belforth Iván Vera Ganchozo

\begin{tabular}{|c|c|}
\hline $\begin{array}{c}\text { Otras especies de Streptucoccus } \\
\text { Especies de Enterococcus }\end{array}$ & $\begin{array}{l}\text { Bacilos Gram Positivos } \\
\text { Especies de Clostridium }\end{array}$ \\
\hline
\end{tabular}

Fuente: Elaboración Propia (2019)

En respuesta a las consideraciones desarrolladas anteriormente se puede indicar que la existencia de cualquiera de los tipos de apendicitis, trae consigo una actuación médica, cónsona a la sintomatología y por ello, al llegar a la necesidad de cumplir con una intervención quirúrgica, se hace notorio que las condiciones anatomopatológico de la apéndices estima la presencia o no de complicaciones postoperatorias como resultado a su propia etiología y patogenia.

\section{Complicaciones Postoperatorias en Pacientes Intervenidos de Apendicitis Aguda}

Los pacientes adultos mayores constituyen un grupo de alto riesgo, sin embargo, el principal determinante de las complicaciones es la severidad de la apendicitis. En la apendicitis aguda simple, las complicaciones son en general de un 10\%. Con la perforación del apéndice, el rango se incrementa de un $15 \%$ a un $65 \%$. Complicaciones específicas relacionadas a la severidad de la apendicitis incluyen: absceso periapendicular, peritonitis difusa, y obstrucción intestinal. Pacientes con apendicitis avanzada pueden llegar a desarrollar sepsis e inclusive fallo orgánico múltiple que puede llegar a ser mortal si un soporte médico-quirúrgico apropiado. En el periodo postoperatorio, las complicaciones infecciosas agudas incluyen la formación de absceso, fístulas cecales e infecciones de la herida quirúrgica. La formación de abscesos intraabdominales en el periodo postoperatorio es mucho más común en apendicitis perforadas. El determinante más importante de la infección de la herida quirúrgica es la severidad de la contaminación al tiempo de la cirugía.

Los antecedentes personales influyen en la evolución de la apendicitis, la mayoría de las publicaciones relacionadas con los abscesos de la pared abdominal y con la fasceitis necrotizante secundarios a apendicitis han sido reportadas en pacientes diabéticos y el diagnóstico de apendicitis aguda a menudo es obstaculizado por síntomas inexpresivos oscurecidos por una enfermedad crónica intercurrente, como la diabetes mellitus. Complicaciones postoperatorias: Es 


\section{Complicaciones postoperatorias en los pacientes sometidos a cirugía de apendicitis aguda}

Vol. 3, núm. 3., (2019)

Jhon Andrés González Macas; Evelyn Adriana Rugel Zerna; Pierina Yaritza Casa Gómez; Katherine Estefania Bajaña Morán; José Luís Moncada Santillán; Belforth Iván Vera Ganchozo

cualquier alteración respecto al curso previsto en la respuesta local y sistémica del paciente quirúrgico. Su etiología puede ser secundaria a la enfermedad primaria, secundaria a la intervención quirúrgica, o secundaria a otros factores no relacionados.

Cuadro $\mathbf{N}^{\circ} 2$ Complicaciones Posoperatorias por Apendicitis Aguda

\begin{tabular}{|l|c|c|}
\hline \multicolumn{1}{|c|}{ Complicaciones Postoperatorias } & No. & \multicolumn{1}{c|}{$\%$} \\
\hline \multicolumn{2}{|c|}{ De la pared } \\
\hline Infección del sitio operatorio (herida) & 90 & 16,1 \\
\hline Dehiscencia de la herida & 22 & 3,9 \\
\hline Evisceración & 1 & 0,2 \\
\hline & Gastrointestinales \\
\hline Ileo paralítico & 15 & \\
\hline Dilatación aguda gástrica & 1 & 2,7 \\
\hline Oclusión intestinal por bridas & 1 & 0,2 \\
\hline Absceso subfrénico derecho & 2 & 0,2 \\
\hline Absceso del saco de Douglas & 1 & 0,3 \\
\hline Abscesos múltiples interasas & 1 & 0,2 \\
\hline
\end{tabular}

Fuente: Elaboración Propia (2019)

Es importante acotar que, las complicaciones registradas en los pacientes intervenidos por una apendicitis aguda, se eleva a medida que aparecen los estadios más graves de esta afección, pues el 10,6 \% de los enfermos con apendicitis aguda temprana sufrió complicaciones, así como también el 14,7 \% de los que presentaron la forma supurada y el 43,8 y el 75,8 \% de los que tenían las variedades gangrenosa y perforada, respectivamente. De allí, que dichas complicaciones tienen lugar en cerca del 20,0 \% de los pacientes. En eta dirección, Patiño (2016), precisa una serie de complicaciones entre las cuales se encuentran las siguientes:

Las infecciones del sitio quirúrgico y las infecciones del espacio profundo o abscesos: son las complicaciones más frecuentes que se observan después de la apendicectomía. Aproximadamente el 5\% de los pacientes con apendicitis no complicada desarrollan infecciones en la herida tras una apendicectomía abierta. La apendicectomía laparoscópica conlleva una incidencia menor de 


\section{Complicaciones postoperatorias en los pacientes sometidos a cirugía de apendicitis aguda}

Vol. 3, núm. 3., (2019)

Jhon Andrés González Macas; Evelyn Adriana Rugel Zerna; Pierina Yaritza Casa Gómez; Katherine Estefania Bajaña Morán; José Luís Moncada Santillán; Belforth Iván Vera Ganchozo

infecciones de la herida; esta diferencia es aún mayor en los pacientes con apendicitis perforada (14 frente a 26\%). Los pacientes con fiebre, leucocitosis y una herida de aspecto normal tras la apendicetomía deben someterse a una TC o una ecografía para descartar la posibilidad de un absceso intraabdominales. Asimismo, si al inspeccionar la herida se comprueba que emana pus por una abertura aponeurótica, se

La infección del sitio operatorio es la complicación más frecuente de la apendicectomía, la que ocurre en 30,0 a 50,0 \% de los intervenidos, seguida de los abscesos intraabdominales y de las gastrointestinales (íleo paralítico posoperatorio). Cabe agregar, en cuanto a las infecciones de la pared fueron las de mayor frecuencia, encabezadas por la incisional superficial y la dehiscencia de la herida quirúrgica, seguidas del íleo paralítico, la infección urinaria y la bronconeumonía en ese orden.

De igual manera, la infección del sitio operatorio depende de muchos factores, entre los que se citan: la virulencia del germen causal, respuesta del paciente a la infección, habilidad del cirujano, criterio para determinar que existe una infección, así como el estado del apéndice en el acto quirúrgico, esta complicación abarca desde el 5,0 \% para las apendicitis tempranas hasta el 75,0 $\%$ de las perforadas.13,14 No obstante, el 4,4\% de los pacientes, con infección del sitio quirúrgico tenían apendicitis agudas tempranas; el 13,7, 30,8 y $37,8 \%$, supuradas, gangrenosas y perforadas, respectivamente.

En las apendicitis agudas sin perforación en 5\% de casos hay complicaciones. En las apendicitis agudas con perforación las complicaciones se presentan en un 30\% de casos. Habitualmente el curso postoperatorio de una apendicitis aguda sin perforación es de una evolución sin sobresaltos y hay una enorme diferencia con la recuperación tormentosa que acompaña a la extirpación de un apéndice perforado con gangrena y con peritonitis; lo cual resalta la importancia del diagnóstico y tratamiento tempranos.

Por lo tanto, las infecciones de la herida son causadas por abscesos locales en la Herida operatoria por gérmenes fecales principalmente Bacteroides frágiles, a los que siguen en frecuencia aerobios Gram (-); Klebsiela, Enterobacter, E. coli. Los signos de infección; dolor, tumor, calor y rubor 


\section{Complicaciones postoperatorias en los pacientes sometidos a cirugía de apendicitis aguda}

Vol. 3, núm. 3., (2019)

Jhon Andrés González Macas; Evelyn Adriana Rugel Zerna; Pierina Yaritza Casa Gómez; Katherine Estefania Bajaña Morán; José Luís Moncada Santillán; Belforth Iván Vera Ganchozo

quizás no se encuentren. Los signos iniciales son dolor excesivo y además molesto alrededor de la herida operatoria de infección local. Si se presentan estos signos deben abrirse de inmediato piel y tejidos subcutáneos. No debe esperarse salida de pus, pues sólo conforme se licúa la grasa necrosada aparece pus. Ninguna herida, sea operatoria o de otra naturaleza, está libre de microorganismos

En la infección de la herida operatoria las bacterias, no sólo están presentes en ella, sino que se multiplican, producen fenómenos tisulares locales y, a menudo, respuesta sistémica. Las mismas no son uniformemente evaluadas: lo que un cirujano titula infección el otro lo niega. Como infección de la herida operatoria deben considerarse las manifestaciones de inflamación con calor, enrojecimiento, edema y dolor (cefalitis) y no solo la constatación de un drenaje purulento por los labios de la incisión. El diagnóstico de infección en el sitio de entrada del catéter se hace por sospecha en un paciente con catéter permanente, en el cual se desarrolla un cuadro séptico sin ninguna otra causa manifiesta. Puede o no haber signos locales de infección. La aspiración y cultivo de la sangre del catéter puede manifestar gran cantidad de gérmenes si el catéter es la fuente de la infección. El tratamiento consiste en extraer el catéter y limpiar localmente la herida, la mayoría de veces con esto es suficiente. Si hay signos de infección general debe instituirse tratamiento con antibióticos contra el germen causante.

Intraabdominales: Suelen deberse a contaminación de la cavidad con microorganismos que escapan del apéndice gangrenoso o perforado, también, pero con menor frecuencia es debido a derrame transoperatorio. El absceso se manifiesta por fiebre héctica o en agujas, con fiebre, malestar y anorexia recurrente. Los abscesos pélvicos producen diarrea y pueden ser palpados al tacto vaginal o rectal; en algunos casos drena espontáneamente a vagina o recto. El absceso subfrénico se puede diagnosticar por signos de derrame al tórax suprayacente e inmovilidad del diafragma afectado. El absceso intraabdominal puede requerir laparotomía exploratoria para su diagnóstico. Todos los abscesos deben ser drenados.

Los abscesos intraperitoneales son la resultante de una contaminación local del peritoneo o de una infección peritoneal generalizada que ha logrado localizarse. La causa de tal infección nace de un 


\section{Complicaciones postoperatorias en los pacientes sometidos a cirugía de apendicitis aguda}

Vol. 3, núm. 3., (2019)

Jhon Andrés González Macas; Evelyn Adriana Rugel Zerna; Pierina Yaritza Casa Gómez; Katherine Estefania Bajaña Morán; José Luís Moncada Santillán; Belforth Iván Vera Ganchozo

proceso inflamatorio previamente instalado en otro sitio del abdomen (absceso subfrénico complicando una apendicitis operada) o de la contaminación peritoneal por cirugía gastro-biliointestinal. Su localización usual es en la pelvis, en áreas subhepáticas o subdiafragmáticas o entre las asas de intestino delgado y el grueso. Se desarrollan 5 o 7 días o más después de la contaminación peritoneal. Su presencia puede ser sospechada por aumento de fiebre, leucocitosis y neutrofilia. La localización pélvica se revela por el desarrollo de una masa dolorosa en el fondo de saco de Douglas; las otras localizaciones pueden ser insidiosas u oscuras y sólo un cuidadoso examen físico con adecuadas investigaciones radiológicas y teniendo presente la posibilidad de su ocurrencia pueden efectuar un diagnóstico temprano. En consecuencia, una tasa de abscesos intraabdominales postoperatorios del $1,1 \%$, que no sugiere un aumento dramático o de importancia clínica en las complicaciones infecciosas.

Fístula Cecal o Estercorácea. La misma puede deberse, por: Retención de Cuerpo Extraño como grasa o dressing. Puntos muy apretados. Ligadura deficiente del muñón apendicular que se deslizó sin haberse invertido - Erosión de la pared del ciego por un dren. Obstrucción del Colon por Neoplasia no descubierta. Retención de una porción apendicular. Enteritis Regional. La mayor parte de Fístulas cierran espontáneamente, todo lo que se requiere es que el trayecto se conserve abierto, hasta que se suspenda el drenaje. Las fístulas fecales no se cierran espontáneamente, si queda punta de apéndice, cuerpo extraño o si el intestino está obstruido distal a la fístula o si la mucosa del intestino quedó en continuidad con la piel. En estos casos el cierre de la fístula requiere operación.

Acumulación de Suero: Es frecuente en heridas que tienen un gran espacio muerto como las de las mastectomías o la de los pacientes obesos. Se las trata mediante la aspiración, o si es voluminosa, mediante una herida por transfixión en el punto más declive aplicando luego un dren con un apósito de compresión. La evacuación debe hacerse lo más precozmente, que con frecuencia son asiento de infección.

Dehiscencia de la Herida: La frecuencia de esta complicación varía mucho en función de cirujano, paciente y tipo de operación. En una herida abdominal, la dehiscencia total produce evisceración 


\section{Complicaciones postoperatorias en los pacientes sometidos a cirugía de apendicitis aguda}

Vol. 3, núm. 3., (2019)

Jhon Andrés González Macas; Evelyn Adriana Rugel Zerna; Pierina Yaritza Casa Gómez; Katherine Estefania Bajaña Morán; José Luís Moncada Santillán; Belforth Iván Vera Ganchozo

y la dehiscencia parcial profunda conduce a la eventración. En la mayoría de casos de dehiscencia, se encuentra que los puntos han desgarrado el tejido. Posiblemente el error más frecuente que condiciona una dehiscencia sea el tensionar demasiado los puntos que van a estrangular los bordes suturados comprometiendo la circulación en esta zona, asimismo los puntos flojos o mal anudados, demasiados puntos, toma de muy poco tejido o material de sutura inapropiado, son otras causas de dehiscencia.

Senos de las líneas de sutura: Cuando el material de sutura no absorbible se infecta, se forma el llamado granuloma a cuerpo extraño, que se abre camino hacia la superficie y después de formar una pequeña tumoración fluctuante, drena material mucopurulento, estableciéndose de esa manera seno o senos en cuyo fondo se encuentra el material de sutura referido, que a veces es expulsado espontáneamente pero que la mayor parte de veces, requiere la introducción de una pinza de punta fina para explorar y extirparlo. De persistir el seno es preferible abrir la porción de herida comprometida, extraer todo el material de sutura que se encuentra en ese lugar y dejar la herida abierta para cierre por segunda intención.

Síndrome infeccioso Focal: Se produce cuando una asociación de gérmenes bacterianos, cocos grampositivos o gramnegativos o bien colonias monobac-terianas: estreptococos, estafilococos, piociánicos, aerobacter, proteus, etc. desencadenan la supuración de la herida operatoria. A esto se puede agregar las micopatías como simple asociación, o asociación más infección micótica: cándidas, aspergilus mисоr. La etiología de la infección del lecho quirúrgico tiene relación, en la mayoría de los casos con el tipo de intervención y órgano o sistema afectado.

Piliflebitis o Piema Portal: Es una enfermedad grave caracterizada por ictericia, escalofrío y fiebre elevada. Se debe a septicemia del sistema venoso portal con desarrollo de abscesos hepáticos múltiples. La piliflebitis acompaña a la apendicitis gangrenosa o perforada y puede aparecer en el pre o postoperatorio. El germen más frecuente es el E. Coli. En la actualidad con el uso de los antibióticos en el pre y postoperatorio su presentación es rara. 


\section{Complicaciones postoperatorias en los pacientes sometidos a cirugía de apendicitis aguda}

Vol. 3, núm. 3., (2019)

Jhon Andrés González Macas; Evelyn Adriana Rugel Zerna; Pierina Yaritza Casa Gómez; Katherine Estefania Bajaña Morán; José Luís Moncada Santillán; Belforth Iván Vera Ganchozo

Íleo Paralítico o Adinámico: En las primeras 24 horas se espera la presencia de un íleo reflejo debido al espasmo producido por la manipulación y cuya resolución es en el postoperatorio inmediato. Sin embargo, puede persistir como resultado de una peritonitis generalizada o una apendicitis complicada; su resolución es lenta y el tratamiento de orden médico: Hidratación con reposición de electrolitos, sonda nasogástrica y antibióticos específicos. Se debe realizar una observación cuidadosa del paciente para detectar una obstrucción mecánica como resultado de adherencias postoperatorias tempranas que sí requerirían tratamiento quirúrgico de emergencia.

Dehiscencia del Muñón Apendicular: Se puede presentar desde el $2^{\circ} \mathrm{o} 3^{\circ}$ día, y puede ser debido a ligadura inadecuada del muñón, o por la administración indebida de un enema evacuante que distiende el intestino y hace que se rompa en el punto más débil, por ello nunca se debe indicar enemas luego de cirugía abdominal. El tratamiento inmediato es laparotomía exploradora y cecostomía con antibióticos específicos.

Cada una de las complicaciones antes citadas, permite resaltar la importancia que tiene una actuación oportuna del médico, pues, mediante su intervención se logran reducir el incremento de las complicaciones, además, garantiza la respectiva mejoría del paciente en un menor tiempo; como se ha destacado, los avances tecnológicos se convierten en herramientas significativas para el desarrollo de cambios progresivos; pero las condiciones anatomapatológicas del pacientes, son factores esenciales para generar aquellas complicaciones generales.

\section{Conclusiones.}

El reportaje referencial llevado a cabo, permite indicar una continuación las respectivas conclusiones que sirven de apoyo reflexivo al trabajo escrito del investigador, entre ellas se citan las siguientes:

La apendicetomía por apendicitis aguda es la operación de urgencia más común en los servicios quirúrgicos, pero no está exenta de complicaciones condicionadas por factores poco conocidos, cuya determinación podría disminuir la morbilidad y mortalidad por esta causa. Fue objetivo de 


\section{Complicaciones postoperatorias en los pacientes sometidos a cirugía de apendicitis aguda}

Vol. 3, núm. 3., (2019)

Jhon Andrés González Macas; Evelyn Adriana Rugel Zerna; Pierina Yaritza Casa Gómez; Katherine Estefania Bajaña Morán; José Luís Moncada Santillán; Belforth Iván Vera Ganchozo

esta investigación identificar algunos factores relacionados con la aparición de complicaciones en los pacientes apendicectomizados por apendicitis aguda.

Por ello, el diagnóstico precoz de la enfermedad y la apendicetomía inmediata con una técnica quirúrgica adecuada previenen la aparición de complicaciones posquirúrgicas y determinan el éxito del único tratamiento eficaz contra la afección más común que causa el abdomen agudo, cuyo pronóstico depende en gran medida y entre otros factores, del tiempo de evolución preoperatoria y de la fase en que se encuentre el proceso morboso al realizar la intervención.

En razón de lo anterior, se puede resaltar que, la apendicetomía por apendicitis aguda representa en estos momentos una intervención quirúrgica urgente frecuente, su práctica clínica, es realizada por el cirujano cuyo diagnóstico, aunque puede apoyarse en exploraciones complementarias (laboratorio, ecografía y tomografía axial computarizada), entre otros. Aunque la población que se interviene por esta enfermedad es mayoritariamente joven y con escasa comorbilidad, también puede presentarse en pacientes de edad avanzada con enfermedades asociadas de riesgo. Es un acto quirúrgico que no debe considerarse menor, a pesar de su brevedad y sencillez de su ejecución en la mayoría de los casos. La posibilidad de desarrollo de complicaciones postoperatorias no es irrelevante, pues, se trata de un proceso séptico intraperitoneal. Sin embargo, los retrasos durante el diagnóstico o la aplicación del tratamiento condicionan la presentación de apendicitis evolucionadas con peritonitis difusa o localizada.

Es importante destacar que cada una de las complicaciones postoperatorias, se encuentra determinadas por las condiciones anatomopatológicas de los pacientes, pues, al tener o presentar alguna enfermedad como diabetes o ser obeso, se incrementan los niveles de dificultad y con ello disminuyen potencialmente las capacidades de respuesta fisiológica del cuerpo. Por ello, al momento de efectuar el respectivo proceso quirúrgico, se hace inminente la necesidad de tener un equipo médico competente que reduzca la presencia de microorganismos o bacterias que puedan afectar a la herida.

Asimismo, se puede ampliar que el tipo de técnica quirúrgica aplicada en la apendicitis aguda con mayor frecuencia en nuestro estudio fue la apendicectomía laparoscópica (AL) en relación a la vía 


\section{Complicaciones postoperatorias en los pacientes sometidos a cirugía de}

apendicitis aguda

Vol. 3, núm. 3., (2019)

Jhon Andrés González Macas; Evelyn Adriana Rugel Zerna; Pierina Yaritza Casa Gómez; Katherine Estefania Bajaña Morán; José Luís Moncada Santillán; Belforth Iván Vera Ganchozo

convencional. Logra ofrecer resultados opuestos, esta diferencia permite inferir que los cambios tecnológicos agregados en la medicina, hacen posible reducir considerablemente las complicaciones en pacientes intervenidos por apendicitis aguda. De allí, la importancia que posee la planificación del tratamiento pre y postoperatorio basado en antibióticos de última generación, con el fin de combatir la posible presencia de bacterias o microorganismos que, al encontrarse en la herida, puedan generar su respectiva infección.

\section{Bibliografía.}

Arroyo, M. (2017). Factores de Riesgos y Complicaciones en Apendicitis Aguda . Salud , 25-39.

Medina, V. (2017). Uso del Drenaje después de una Apendicectomía Abierta . Cocharne, 11-22.

Méndz, G. (2018). Metodología e Investigación . México: Mac Graw Hill .

Mogollón, N. (2018). Técnicas para la Investigación Documental . Barquisimeto : CIDEG.

Patiño, J. (2016). Complicaciones de la Apendicectomía . Clínica Quirúrgica , 66-78.

Sample, G. (2015). Complicaciones Infecciosas después de la Apendicectomía Laparoscopica . IntraMed, 8-15.

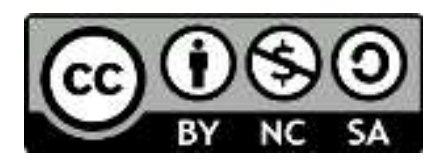

RECONOCIMIENTO-NOCOMERCIAL-COMPARTIRIGUAL

CC BY-NC-SA

ESTA LICENCIA PERMITE A OTROS ENTREMEZCLAR, AJUSTAR Y CONSTRUIR A PARTIR DE SU OBRA CON FINES NO COMERCIALES, SIEMPRE Y CUANDO LE RECONOZCAN LA AUTORÍA Y SUS NUEVAS CREACIONES ESTÉN BAJO UNA LICENCIA CON LOS MISMOS TÉRMINOS. 